\title{
O Impacto da utilização de bundles na prevenção da pneumonia associada à ventilação mecânica: uma revisão integrativa
}

\author{
The impact of the use of bundles in the prevention of pneumonia \\ associated with mechanical ventilation: an integrative review
}

\author{
Francieli Tozatti Ficagna' $\bullet$ Rafaela Vivian Valcarenghi ${ }^{2} \bullet$ Nicoli Ferreira Demarche $^{3} \bullet$ Diego Ferreira $^{4}$
}

\begin{abstract}
RESUMO
Introdução: Bundle é um conjunto de medidas realizadas de forma associada capaz de contribuir para a prevenção de diversas complicações decorrentes da pneumonia associada à ventilação mecânica (PAV) no ambiente da Unidade de Terapia Intensiva (UTI). Objetivo:Verificar qual o impacto da utilização de bundles na prevenção da PAV. Métodos: Estudo de revisão integrativa da literatura nas bases de dados LILACS e PubMed, nos meses de fevereiro e março de 2019. Resultados: Foram analisados 33 artigos, sendo que a maioria aponta para redução das taxas de PAV e 18 destes apresentam taxas menores que 10 episódios de PAV/I000 dias de ventilação mecânica durante a intervenção.A utilização do bundle também se mostrou capaz de promover mudanças na diminuição do tempo de internação, do uso da ventilação mecânica, na redução dos custos hospitalares e diminuição da mortalidade. Conclusões: $O$ bundle sugerido pelo Institute for Healthcare Improvement possui medidas que quando aplicadas provocam melhorias para os pacientes, equipe e instituição de saúde.A modificação de tal ferramenta por parte das unidades hospitalares, a fim de adaptá-la para o seu cenário, também se mostra eficaz.A dificuldade de adesão às medidas por parte dos profissionais traz a necessidade de estudos para buscar compreender quais os obstáculos mais presentes através da perspectiva da equipe.

Palavras-chave: Bundle; Cuidados críticos; Pneumonia associada à ventilação mecânica; Cuidados de enfermagem.
\end{abstract}

\begin{abstract}
Introduction: Bundle is a set of measures performed in an associated way capable of contributing to the prevention of several complications resulting from pneumonia associated with mechanical ventilation (VAP) in the Intensive Care Unit (ICU) environment. Objective:To verify the impact of the use of bundles to prevent pneumonia associated with mechanical ventilation (VAP). Methods: Study of integrative literature review in the LILACS and PubMed databases, in the months of February and March 2019. Results: A total of 33 articles were analyzed, most of which point to a reduction in the rates of VAP, and 18 of these have rates lower than 10 episodes of VAP / 1000 days of mechanical ventilation during the intervention The use of the bundle was also able to promote changes in reducing the length of hospital stay, the use of mechanical ventilation, reducing hospital costs and decreasing mortality. Conclusions: The bundle suggested by the Institute for Healthcare Improvement has measures that when applied lead to improvements for patients, staff, and health facility. The modification of such a tool by the hospital units, in order to adapt it to their scenario, is also effective. The difficulty of adherence to the measures on the part of the professionals brings the need of studies to try to understand what obstacles present more through the perspective of the team.
\end{abstract}

Keywords: Bundle; Critical care; Pneumonia associated with mechanical ventilation; Nursing care.

I Mestre em Saúde Coletiva/Epidemiologia pela Universidade Federal de Santa Catarina - UFSC. Especialista em Cardiologia e Hemodinâmica. Enfermeira Efetiva da Unidade de Pronto Atendimento UPA- 24 h do Município de Biguaçu.

2 Doutora em Enfermagem pela Universidade Federal de Santa Catarina - UFSC. Docente da Universidade Estácio de Santa Catarina-Campus- São José.

3 Enfermeira Residente em Saúde da Família pela Escola de Saúde Pública do Município de Florianópolis-SC.

4 Enfermeiro da Secretaria de Saúde do Estado de Santa Catarina e da Clínica TOP MED no Município de Florianópolis-SC. 


\section{INTRODUÇÃO}

As infecções relacionadas à assistência à saúde (IRAS) são de grande frequência em unidades hospitalares ${ }^{(1)}$. Dentre elas, se destaca a pneumonia, causada pela infiltração de um microrganismo patogênico (como vírus e bactérias) no parênquima pulmonar. Considera-se como pneumonia associada à ventilação mecânica (PAV), quando a mesma ocorre entre 48 a 72 horas após a intubação endotraqueal e início da ventilação mecânica invasiva, não devendo o agente infeccioso estar presente no momento da admissão hospitalar ${ }^{(2-3)}$. O surgimento da pneumonia se dá, comumente, devido à perda de barreiras protetoras e da mobilidade (impedindo movimentos de deglutição e tosse, por exemplo), favorecendo o desenvolvimento de microrganismos ${ }^{(4-5)}$.

O uso da ventilação mecânica (VM) se faz presente, principalmente em Unidades de Terapia Intensiva (UTI), onde os pacientes apresentam quadros clínicos mais graves e pela maior necessidade de suporte ventilatório. $A$ atuação da VM se dá através do emprego de uma pressão positiva ou negativa nas vias aéreas, assim corrigindo insuficiências respiratórias, apneias, ou até mesmo contribuindo para a diminuição do esforço respiratório. Infelizmente, em algumas situações, a VM pode trazer prejuízos à vida do paciente, como a pneumonia, barotrauma, alterações hemodinâmicas e podendo levar à morte ${ }^{(4)}$.

Avaliando o índice de mortalidade dos pacientes ventilados, o Institute for Healthcare Improvement $(\mathrm{IHI})$ desenvolveu o bundle de ventilação, que é um conjunto de medidas baseadas em evidências científicas, que quando realizadas de forma associada contribuem para a prevenção de diversas complicações que os pacientes em uso de ventilação mecânica desenvolvem, como: sangramento gastrointestinal induzido por estresse, tromboembolismo venoso e, inclusive, a pneumonia associada à ventilação mecânica. Os elementos que foram identificados para compor o bundle foram: elevação da cabeceira da cama entre 30 e $45^{\circ}$, profilaxia da úlcera péptica, profilaxia da trombose venosa profunda (exceto em casos de contraindicação) e higiene bucal com Clorexidina oral a 0,12\%. Com estas intervenções foi constatado uma diminuição nas taxas de mortalidade em locais em que o bundle foi aplicado(6).

Desde então, outros bundles de ventilação vem sendo propostos $^{(7-9)}$, com sugestões de medidas diferentes para implementar. Entretanto, cabe ressaltar, que o Institute for Healthcare Improvement recomenda que não sejam desenvolvidos bundles muito extensos, devido à maior dificuldade em gerenciá-los e aplicá-los ${ }^{(6)}$.

É importante destacar que a PAV apresenta uma alta taxa de mortalidade, além de contribuir para o aumento do tempo de internação e consequentemente maiores custos hospitalares, levando a um maior impacto econômico para os sistemas de saúde público e privado.
Além disso, ressalta-se os prejuízos à vida do paciente que demanda mais tempo em um leito de UTI, como as mudanças drásticas nos hábitos de vida, a separação de seus familiares devido ao pouco tempo de visita no setor e os sentimentos negativos como angústia, ansiedade e insegurança. Portanto, o processo de adoecimento é vivenciado de forma intensa não só pelo paciente, mas também pelo seu núcleo familiar, que remaneja sua rotina para acompanhar de perto o tratamento de seu ente querido. Em relação à incidência da PAV, existem dados divulgados no Estado de São Paulo, em 2015, relatando 9,87 casos por 1.000 dias de uso de ventilador em UTI adulto, 13,40 casos por 1.000 dias de uso de ventilador em hospitais de ensino e 6,56 casos em hospitais privados. Em relação à mortalidade mundial da PAV, a mesma pode variar entre $20 \%$ a $60 \%(10-11)$.

No que tange ao papel dos profissionais na prevenção da PAV, a equipe de enfermagem possui grande protagonismo, pois realiza muitos cuidados relacionados à ventilação mecânica, tais como: aspiração de secreção de vias aéreas, higiene oral, posicionamento da cabeceira do leito, entre outros ${ }^{(12-13)}$.

Conforme a Lei $n^{\circ} 7.498 / 1986$, do exercício profissional da enfermagem, cabe especificamente ao enfermeiro “o planejamento, organização, coordenação, execução e avaliação dos serviços da assistência de enfermagem"(14). Portanto, o enfermeiro deve atuar, através destas atribuições, baseando-se em conhecimento científico, e educando a sua equipe quanto à realização correta de técnicas assépticas, a fim de prevenir não só a PAV, mas também outras infecções relacionadas à assistência à saúde. No ambiente de uma UTI, estas responsabilidades se intensificam, visto que além das atividades relacionadas à organização e coordenação do serviço, o enfermeiro enfrenta em sua rotina demandas assistenciais complexas, visto o grau de adoecimento dos pacientes presentes neste setor ${ }^{(15)}$.

A justificativa da temática se resume em virtude da alta taxa de incidência da PAV e seus agravantes, além da necessidade de atualização constante por parte dos profissionais, que se encontram em contato direto e contínuo com o paciente através de uma assistência adequada e segura que podem minimizar os riscos de ocorrência desta patologia. Por isso, é imprescindível conhecer as ferramentas mais adequadas para minimizar ou diminuir os riscos de PAV.

Assim sendo, o objetivo deste estudo foi verificar qual o impacto da utilização de bundles na prevenção da pneumonia associada à ventilação mecânica.

\section{MÉTODOS}

Trata-se de um estudo de revisão integrativa da literatura. Durante o desenvolvimento do estudo foram respeitadas as seis etapas preconizadas: identificação do 
tema e seleção da hipótese ou questão de pesquisa; estabelecimento de critérios para inclusão e exclusão de estudos/amostragem ou busca na literatura; definição das informações a serem extraídas dos estudos selecionados/ categorização dos estudos; avaliação dos estudos incluídos; interpretação dos resultados; e apresentação da revisão/síntese do conhecimento(17-18). Estipulou-se a seguinte questão norteadora: qual o impacto da utilização de bundles na prevenção da pneumonia associada a ventilação mecânica?

O levantamento bibliográfico foi realizado nos meses de fevereiro e março do ano de 2019, nas bases de dados National Library of Medicine and National Institutes of Health (PubMed) e Literatura Latino-Americana e do Caribe em Ciência da Saúde (LILACS). A base de dados Scientific Eletronic Library Online (SciELO) também foi consultada, porém não foram encontradas publicações diferentes das já selecionadas. Os descritores utilizados foram "Bundle AND pneumonia AND cuidados críticos" e "Bundle AND pneumonia AND cuidados de enfermagem" e "bundle AND pneumonia AND ventilação mecânica"; "Bundle AND pneumonia AND critical care" e "Bundle AND pneumonia AND nursing care" e "bundle AND pneumonia AND mechanical ventilation”; " Bundle AND neumonía AND cuidado critico" e "Bundle AND neumonía AND cuidados de enfermería" e "bundle AND neumonía AND ventilación mecânica".

Foram estabelecidos os seguintes critérios de inclusão: artigos científicos disponíveis em texto completo e gratuito nas bases consultadas, publicados nos idiomas inglês, português e espanhol e que descrevessem a implementação de um bundle com o objetivo de prevenir a pneumonia associada à ventilação mecânica, apresentando o resultado de seu impacto. A fim de ampliar a pesquisa, buscou-se em todos os anos de publicação, não sendo realizado recorte temporal. Foram critérios de exclusão: tipo de publicação (cartas, editoriais) ou que apresentassem somente resumo, artigos duplicados, artigos de revisão, dissertações, teses e estudos que não respondessem ao objetivo traçado. Foram descartados também, estudos em que houveram modificações no bundle durante o período de avaliação de seu impacto, ou que não apresentaram dados de forma clara sobre o período anterior e posterior à implementação das intervenções.

Tratando-se da análise dos dados, os artigos selecionados para revisão foram organizados em um instrumento de coleta de dados, estruturado em autor, ano de publicação, país, título, periódico, Qualis-CAPES, tipo de estudo, objetivos e resultados do impacto dos bundles de prevenção da pneumonia associada à ventilação mecânica. As análises feitas em concordância entre os pesquisadores, foram aceitas imediatamente, no entanto, quando havia discordância, eram discutidos caso a caso até chegar em comum acordo entre estes. Os dados foram analisados de forma descritiva.

\section{RESULTADOS}

$\mathrm{Na}$ pesquisa realizada n0 PubMed, foram disponibilizados 216 publicações, sendo 85 em texto completo e gratuito. Após realizar leitura do título e resumo, e, concomitantemente aplicar os supracitados critérios de inclusão e exclusão, 54 destes foram descartados e 31 selecionados. Já na busca realizada no LILACS, encontrou-se 9 publicações. Destas, somente dois encaixavamse nos critérios estipulados. Quanto ao idioma o mais prevalente foi o inglês, totalizando 30 publicações, seguido do português com três publicações restantes. De acordo com os artigos selecionados, os anos de publicação sobre a temática, variaram de 2010 a 2018.

No Quadro I, são apresentadas as informações de cada artigo que compuseram a presente revisão. Através do mesmo, constata-se que a maioria dos estudos apontou para redução das taxas de pneumonia associada à ventilação mecânica, e, 18/33 apresentaram taxas menores que 10 episódios de $\mathrm{PAV} / \mathrm{I} 000$ dias de ventilação mecânica durante a intervenção.

\section{DISCUSSÃO}

Por meio da análise dos estudos, é possível observar a redução das taxas de PAV através da utilização de uma abordagem multidimensional (implementação do bundle acompanhado de ações como educação da equipe, acompanhamento dos resultados e feedback aos profissionais). Através da mesma, observa-se diminuição de $39 \%$ nas taxas de PAV em hospitais da Arábia Saudita (7,84 para 4,74 por 1000 dias VM) ${ }^{(18)}$. Essa taxa pode ser comparada com implementações da mesma ferramenta em outros países, com redução de $31 \%$ na Argentina (5I,28 vs. 35,50$)^{(51)}, 76 \%$ na China $(24, I \text { vs. } 5,7)^{(52)}, 71 \%$ em Cuba (52.63 vs. 15.32$)^{(41)}, 46 \%$ na Turquia (3I.I4 vs. 16.82$)^{(53)} \mathrm{e}$ $38 \%$ na Índia (17.43 vs. I0.8I) $)^{(42)}$. Reforçando estes dados, as medidas aplicadas nos estudos avaliados têm sido associadas a treinamentos dos profissionais, que tiveram aderência, principalmente dos enfermeiros ${ }^{(19)}$. A educação do enfermeiro reflete diretamente na execução das estratégias de prevenção ${ }^{(24)}$, visto que muitas delas fazem parte dos cuidados de enfermagem ${ }^{(54)}$.

O acompanhamento e feedback aos profissionais de saúde, também é relevante, como mostram Concanour e col. $(2006)^{(55)}$, onde os casos de PAV só diminuíram depois que a adesão com o bundle foi auditada diariamente com feedback semanal concedido aos profissionais ${ }^{(55)}$. Por isso, diferenças não relevantes entre grupos com e sem implementação do bundle podem estar relacionadas a essas limitações, quando a adesão da equipe não é formalmente estudada durante a intervenção(20). 
QUADRO 1 - Artigos incluídos na revisão integrativa conforme suas características. São José, SC, Brasil, 2019

\begin{tabular}{|c|c|c|c|c|c|}
\hline $\begin{array}{l}\text { Autor/Anol } \\
\text { País }\end{array}$ & Título & $\begin{array}{l}\text { Periódico/ } \\
\text { Qualis- } \\
\text { CAPES }\end{array}$ & Tipo de estudo & Objetivos & $\begin{array}{c}\text { Resultados do impacto dos } \\
\text { bundles de prevenção da } \\
\text { pneumonia associada à } \\
\text { ventilação mecânica }\end{array}$ \\
\hline $\begin{array}{l}\text { Al-abdely HM, } \\
\text { e col. } \\
2018^{18} \\
\text { Arábia } \\
\text { Saudita }\end{array}$ & $\begin{array}{l}\text { Impact of the } \\
\text { International } \\
\text { Nosocomial } \\
\text { Infection Control } \\
\text { Consortium } \\
\text { (INICC)'s } \\
\text { multidimensional } \\
\text { approach on rates of } \\
\text { ventilator-associated } \\
\text { pneumonia in } \\
\text { intensive care units } \\
\text { in } 22 \text { hospitals of } \\
14 \text { cities of the } \\
\text { Kingdom of Saudi } \\
\text { Arabia }\end{array}$ & $\begin{array}{l}\text { J Infect } \\
\text { Public } \\
\text { Health / } \\
\text { A1 }\end{array}$ & $\begin{array}{l}\text { Multicêntrico, prospectivo, de } \\
\text { coorte. }\end{array}$ & $\begin{array}{c}\text { Analisar o impacto } \\
\text { da Abordagem } \\
\text { Multidimensional } \\
\text { (IMA) do International } \\
\text { Nosocomial Infection } \\
\text { Control Consortium } \\
\text { (INICC) e do uso do } \\
\text { Sistema de Vigilância } \\
\text { Online (ISOS) do INICC } \\
\text { nas taxas de PAV na } \\
\text { Arábia Saudita de } \\
\text { setembro de } 2013 \text { a } \\
\text { fevereiro de } 2017 .\end{array}$ & $\begin{array}{l}\text { Durante a implementação do } \\
\text { programa multidimensional } \\
\text { de controle de infecção, foi } \\
\text { registrada uma média de } \\
\text { uso de VM de } 9,4 \text {. A taxa de } \\
\text { PAV por } 1.000 \text { dias de VM } \\
\text { foi reduzida para } 4,74 \text { no } \\
\text { segundo ano, representando } \\
\text { uma redução acumulada de } \\
39 \% \text { na taxa de PAV. A eficácia } \\
\text { ajustada da intervenção foi de } \\
27 \% \text {. }\end{array}$ \\
\hline $\begin{array}{l}\text { Álvarez- } \\
\text { Lerma F e col. } \\
2018^{19} \\
\text { Espanha }\end{array}$ & $\begin{array}{c}\text { Prevention } \\
\text { of Ventilator- } \\
\text { Associated } \\
\text { Pneumonia: } \\
\text { The Multimodal } \\
\text { Approach of the } \\
\text { Spanish ICU } \\
\text { "Pneumonia Zero" } \\
\text { Program }\end{array}$ & $\begin{array}{l}\text { Crit Care } \\
\text { Med /A1 }\end{array}$ & $\begin{array}{l}\text { Estudo prospectivo, } \\
\text { intervencio- } \\
\text { nista e multicên- } \\
\text { trico. }\end{array}$ & $\begin{array}{c}\text { Avaliar o impacto do } \\
\text { projeto "Pneumonia } \\
\text { Zero" na redução das } \\
\text { taxas de PAV nas UTIs } \\
\text { espanholas. }\end{array}$ & $\begin{array}{c}\text { A taxa de densidade de } \\
\text { incidência de PAV diminuiu } \\
\text { de } 9,83(95 \% \text { IC, } 8,42-11,48) \\
\text { por } 1.000 \text { dias de ventilação } \\
\text { no período de referência para } \\
4,34(95 \% \mathrm{Cl}, 3,22-5,84) \text { após } \\
\text { 19-21 meses de participação. }\end{array}$ \\
\hline $\begin{array}{l}\text { Burja S e col. } \\
\qquad 2017^{20} \\
\text { Eslovênia }\end{array}$ & $\begin{array}{l}\text { Efficacy of a } \\
\text { bundle approach } \\
\text { in preventing } \\
\text { the incidence } \\
\text { of ventilator } \\
\text { associated } \\
\text { pneumonia (VAP) }\end{array}$ & $\begin{array}{l}\text { Bosn J } \\
\text { Basic Med } \\
\text { Sci /B2 }\end{array}$ & Estudo retrospectivo & $\begin{array}{c}\text { Determinar a eficácia do } \\
\text { bundle de prevenção de } \\
\text { PAV implementado na } \\
\text { instituição, avaliando a } \\
\text { incidência de PAV antes } \\
\text { e após a introdução do } \\
\text { bundle. }\end{array}$ & $\begin{array}{c}\text { Os pacientes foram divididos } \\
\text { em dois grupos: no-VAP-B } \\
\text { (55 pacientes submetidos à } \\
\text { VM antes do bundle) e VAP-B } \\
\text { (74 pacientes submetidos a } \\
\text { VM depois das medidas do } \\
\text { bundle). No grupo no-VAP-B, } \\
41,8 \% \text { desenvolveram PAV } \\
\text { (45 ocorrências/ } 1.000 \text { dias } \\
\text { de ventilação) comparado a } \\
25,7 \% \text { dos pacientes no grupo } \\
\text { VAP-B (30 ocorrências/ } 1.000 \\
\text { dias de ventilação). PAV tardia: } \\
\text { grupo VAP-B comparado } \\
\text { ao grupo no- VAP-B (13,5\% } \\
\text { versus } 30,9 \%, p=0,027 \text { ). }\end{array}$ \\
\hline $\begin{array}{l}\text { Su KC e col. } \\
2017^{21} \text { Taiwan }\end{array}$ & $\begin{array}{l}\text { A simplified } \\
\text { prevention bundle } \\
\text { with dual hand } \\
\text { hygiene audit } \\
\text { reduces early- } \\
\text { onset ventilator } \\
\text { associated } \\
\text { pneumonia in } \\
\text { cardiovascular } \\
\text { surgery units: An } \\
\text { interrupted time- } \\
\text { series analysis }\end{array}$ & $\begin{array}{l}\text { Plos One/ } \\
\quad \text { A1 }\end{array}$ & $\begin{array}{c}\text { Estudo quase experimental } \\
\text { de } 3 \text { anos com análise de } \\
\text { série temporal. }\end{array}$ & \begin{tabular}{|} 
Investigar o efeito \\
de um bundle de \\
prevenção simplificado \\
com auditoria dupla \\
de higiene das mãos \\
à base de álcool \\
na incidência de \\
pneumonia associada \\
à ventilação (PAV) \\
precoce.
\end{tabular} & \begin{tabular}{|} 
O estudo foi realizado em \\
4 fases. As incidências de \\
PAV (eventos / 1.000 dias de \\
ventilação) nas fases 1 a 4 \\
foram de $39,1,40,5,15,9$ e \\
20,4 . A PAV foi reduzida em \\
$59 \%$ na fase 3 em comparação \\
à fase 1 , porém sua incidência \\
recuperou-se na fase 4 \\
(vs. fase $1, P=0,078$ ), não \\
conseguindo manter uma \\
melhora significativa como na \\
fase 3 .
\end{tabular} \\
\hline
\end{tabular}




\begin{tabular}{|c|c|c|c|c|c|}
\hline $\begin{array}{l}\text { Haque A, e } \\
\text { col. } \\
2017^{22} \\
\text { Paquistão }\end{array}$ & $\begin{array}{l}\text { Implementation of } \\
\text { Ventilator Bundle in } \\
\text { Pediatric Intensive } \\
\text { Care Unit of a } \\
\text { Developing Country }\end{array}$ & $\begin{array}{l}\text { J Coll } \\
\text { Physicians } \\
\text { Surg Pak/ } \\
\text { A1 }\end{array}$ & $\begin{array}{l}\text { Estudo de coorte, } \\
\text { retrospectivo. }\end{array}$ & $\begin{array}{c}\text { Avaliar a frequência } \\
\text { de PAV após a } \\
\text { implementação rigorosa } \\
\text { do bundle de ventilação } \\
\text { na UTI Pediátrica. }\end{array}$ & $\begin{array}{l}\text { Houve apenas } 4 \text { episódios de } \\
\text { PAV em } 2012 \text { e não houveram } \\
\text { mais casos durante os dois } \\
\text { anos seguintes (2013 e 2014). } \\
\text { A frequência foi, então, de } \\
0,7(4 / 565) \text {. A densidade de } \\
\text { incidência foi de } 1.62 / 1.000 \\
\text { dias de ventilação. }\end{array}$ \\
\hline $\begin{array}{l}\text { Akdogana O } \\
\text { e col. } \\
2017^{23} \\
\text { Turquia }\end{array}$ & $\begin{array}{l}\text { Assessment of the } \\
\text { effectiveness of a } \\
\text { ventilator } \\
\text { associated } \\
\text { pneumonia } \\
\text { prevention bundle } \\
\text { that } \\
\text { contains } \\
\text { endotracheal tube } \\
\text { with subglottic } \\
\text { drainage and } \\
\text { cuff pressure } \\
\text { monitorization }\end{array}$ & $\begin{array}{l}\text { Braz J } \\
\text { Infect Dis. } \\
\text { /B2 }\end{array}$ & $\begin{array}{l}\text { Estudo prospectivo e } \\
\text { controlado. }\end{array}$ & $\begin{array}{c}\text { Avaliar a eficácia do } \\
\text { bundle de PAV contendo } \\
\text { tubo endotraqueal } \\
\text { com drenagem de } \\
\text { secreção subglótica } \\
\text { e monitorização da } \\
\text { pressão do manguito. }\end{array}$ & $\begin{array}{c}\text { A incidência de PAV diminuiu } \\
\text { de } 40,82 \text { para } 22,16 \text { por } 1.000 \\
\text { dias de ventilação entre os } \\
\text { pacientes dos grupos controle } \\
\text { e caso, respectivamente ( } p \\
<0,05) \text {. Em média, a PAV } \\
\text { ocorreu } 17,33 \pm 21,09 \text { dias no } \\
\text { grupo caso e } 10,43 \pm 7,83 \text { dias } \\
\text { no grupo controle }(p=0,04) \text {. }\end{array}$ \\
\hline $\begin{array}{l}\text { Mogyorodi B } \\
\text { e col. } \\
2016^{24} \\
\text { Hungria }\end{array}$ & $\begin{array}{l}\text { Ventilator- } \\
\text { associated } \\
\text { pneumonia and } \\
\text { the importance } \\
\text { of education of } \\
\text { ICU nurses on } \\
\text { prevention - } \\
\text { Preliminary results }\end{array}$ & $\begin{array}{l}\text { Interv Med } \\
\text { Appl Sci / } \\
\text { B3 }\end{array}$ & $\begin{array}{c}\text { Estudo observacional } \\
\text { prospectivo. }\end{array}$ & $\begin{array}{c}\text { Examinar as mudanças } \\
\text { na incidência e no } \\
\text { espectro bacteriano da } \\
\text { PAV após a implantação } \\
\text { de um bundle. A adesão } \\
\text { dos enfermeiros da UTI } \\
\text { também foi estudada. }\end{array}$ & $\begin{array}{l}\text { A incidência de PAV foi de } 21,5 \\
\text { na primeira fase e } 12,0 \text { na } \\
\text { segunda fase (por } 1.000 \text { dias } \\
\text { de ventilação). Houve melhora } \\
\text { significativa na adesão da } \\
\text { elevação da cabeceira do, } \\
\text { higiene bucal, higienização } \\
\text { das mãos, aspiração } \\
\text { endotraqueal e retirada do } \\
\text { condensado do circuito. }\end{array}$ \\
\hline $\begin{array}{l}\text { Rodrigues AN } \\
\text { e col. } \\
2016^{25} \text { Brasil, }\end{array}$ & $\begin{array}{l}\text { Impactos e fatores } \\
\text { determinantes } \\
\text { no bundle de } \\
\text { pneumonia } \\
\text { associada à } \\
\text { ventilação } \\
\text { mecânica. }\end{array}$ & $\begin{array}{l}\text { Rev. Bras. } \\
\text { Enferm. } \\
\text { [online] } \\
\text { /A2 }\end{array}$ & $\begin{array}{l}\text { Estudo longitudinal } \\
\text { retrospectivo, descritivo, com } \\
\text { abordagem quantitativa. }\end{array}$ & $\begin{array}{c}\text { Avaliar os impactos e } \\
\text { fatores determinantes } \\
\text { no cumprimento do } \\
\text { bundle para redução da } \\
\text { pneumonia associada à } \\
\text { ventilação mecânica. }\end{array}$ & $\begin{array}{c}\text { Aumento na incidência de PAV } \\
\text { após implantação do bundle } \\
\text { de } 11,5 \text { para } 16,4 \text { pacientes; } \\
\text { bactérias gram-negativas } \\
\text { presentes. Os óbitos foram }= \\
\text { ou }>50 \% .\end{array}$ \\
\hline $\begin{array}{c}\text { Peña-López Y } \\
\text { e col. } \\
2016^{26} \\
\text { Espanha }\end{array}$ & $\begin{array}{l}\text { Implementing } \\
\text { a care bundle } \\
\text { approach reduces } \\
\text { ventilator- } \\
\text { associated } \\
\text { pneumonia and } \\
\text { delays ventilator- } \\
\text { associated } \\
\text { tracheobronchitis in } \\
\text { children: } \\
\text { differences } \\
\text { according to } \\
\text { endotracheal or } \\
\text { tracheostomy } \\
\text { devices }\end{array}$ & $\begin{array}{l}\text { Int J Infect } \\
\text { Dis/B3 }\end{array}$ & $\begin{array}{l}\text { Estudo de coorte } \\
\text { intervencionista prospectivo. }\end{array}$ & \begin{tabular}{|c|} 
Avaliar se a \\
implementação de um \\
bundle de cuidados \\
com ventilador na \\
UTI Pediátrica (UTIP) \\
poderia reduzir \\
simultaneamente a \\
incidência de PAV \\
e traqueobronquite \\
associada ao ventilador \\
em crianças gravemente \\
doentes, incluindo \\
aquelas em ventilação \\
mecânica prolongada.
\end{tabular} & $\begin{array}{c}\text { Redução na PAV entre } \\
\text { pacientes traqueostomizados } \\
(8,16,3,27 \text { e } 0,65) \text { por } 1.000 \\
\text { dias de VM com traqueostomia } \\
\text { (antes da intervenção, } \\
\text { após a implantação do } \\
\text { bundle geral, e após a } \\
\text { intervenção de traqueostomia, } \\
\text { respectivamente). Redução } \\
\text { da mortalidade na UTIP, de } \\
28,4 \% \text { para } 16,6 \% \text { (risco } \\
\text { relativo 0,58). }\end{array}$ \\
\hline $\begin{array}{l}\text { Parisi M e col. } \\
2016^{27} \text { Grécia }\end{array}$ & $\begin{array}{l}\text { Use of ventilator } \\
\text { bundle } \\
\text { and staff education } \\
\text { to decrease } \\
\text { ventilator } \\
\text { associated } \\
\text { pneumonia in } \\
\text { intensive care } \\
\text { patients }\end{array}$ & $\begin{array}{l}\text { Crit Care } \\
\text { Nurse /B2 }\end{array}$ & $\begin{array}{l}\text { Estudo prospectivo de } \\
\text { intervenção. }\end{array}$ & $\begin{array}{l}\text { Avaliar a incidência de } \\
\text { PAV em uma unidade } \\
\text { multidisciplinar de } \\
\text { terapia intensiva e } \\
\text { examinar os efeitos } \\
\text { da implantação de } \\
\text { bundles de ventilação } \\
\text { e educação da equipe } \\
\text { sobre sua incidência. }\end{array}$ & $\begin{array}{c}\text { Antes e pós-intervenção, a } \\
\text { taxa de PAV diminuiu de } 21,6 \\
\text { para } 11,6 \text { por } 1.000 \text { dias de } \\
\text { ventilação. O tempo de UTI } \\
\text { diminuiu de } 36 \text { para } 27 \text { dias e } \\
\text { a duração da VM diminuiu de } \\
26 \text { para } 21 \text { dias. }\end{array}$ \\
\hline
\end{tabular}




\begin{tabular}{|c|c|c|c|c|c|}
\hline $\begin{array}{c}\text { Ferreira C e } \\
\text { col. } \\
2012^{28} \\
\text { Brasil }\end{array}$ & $\begin{array}{l}\text { The effectiveness } \\
\text { of a bundle in } \\
\text { the prevention } \\
\text { of ventilator- } \\
\text { associated } \\
\text { pneumonia }\end{array}$ & $\begin{array}{l}\text { The Braz J } \\
\text { Infect Dis } \\
\text { IA2 }\end{array}$ & Estudo retrospectivo. & $\begin{array}{c}\text { Avaliar o impacto de um } \\
\text { bundle chamado FAST } \\
\text { HUG na PAV, pesar os } \\
\text { custos dos pacientes } \\
\text { com PAV na UTI e a } \\
\text { mortalidade hospitalar } \\
\text { por PAV. } \\
\text { *FAST HUG é uma } \\
\text { lista de verificação } \\
\text { que destaca os } \\
\text { principais aspectos dos } \\
\text { cuidados gerais para } \\
\text { pacientes graves. A } \\
\text { ajuda mnemônica } \\
\text { enfatiza a importância } \\
\text { das seguintes } \\
\text { práticas clínicas: } \\
\text { alimentação, analgesia, } \\
\text { sedação, profilaxia } \\
\text { tromboembólica, } \\
\text { elevação da cabeceira } \\
\text { do leito, profilaxia da } \\
\text { úlcera por estresse e } \\
\text { controle glicêmico. }\end{array}$ & $\begin{array}{c}\text { Redução nas taxas de PAV } \\
(p<0,01) \text { e de mortalidade ( } p \\
<0,01) \text {. Redução nos custos } \\
\text { hospitalares da UTI ( } p<0,05) \text {. } \\
\text { Não houve diferença de } \\
\text { sobrevida entre os dois grupos } \\
\text { (Sem FAST HUG e com FAST } \\
\text { HUG) nos primeiros } 20 \text { dias } \\
\text { de internação. Os primeiros } \\
80 \text { dias de internação, } \\
50 \% \text { do grupo FAST HUG } \\
\text { sobreviveu, em comparação } \\
\text { com } 20 \% \text { para aqueles sem } \\
\text { a intervenção. Quanto maior } \\
\text { o tempo de permanência, } \\
\text { menor a taxa de sobrevida dos } \\
\text { pacientes sem intervenção. }\end{array}$ \\
\hline $\begin{array}{c}\text { Malcolm D e } \\
\text { col. } \\
2015^{29} \\
\text { Escócia }\end{array}$ & $\begin{array}{l}\text { Details behind the } \\
\text { dots: How different } \\
\text { intensive care units } \\
\text { used common } \\
\text { and contrasting } \\
\text { methods to } \\
\text { prevent ventilator } \\
\text { associated } \\
\text { pneumonia }\end{array}$ & $\begin{array}{l}\text { BMJ Qual } \\
\text { Improv Rep } \\
\text { /B2 }\end{array}$ & Estudo de séries temporais. & $\begin{array}{c}\text { Reduzir a incidência de } \\
\text { pneumonia associada } \\
\text { à ventilação mecânica } \\
\text { (PAV) a zero ou } 300 \\
\text { dias corridos entre os } \\
\text { eventos, através da } \\
\text { implementação de um } \\
\text { bundle de Prevenção de } \\
\text { PAV em duas UTls de } \\
\text { hospitais distintos. }\end{array}$ & $\begin{array}{l}\text { Comparando os períodos } \\
\text { de tempo, antes e depois de } \\
80 \% \text { de adesão ao bundle } \\
\text { de prevenção da PAV, esta } \\
\text { apresentou uma redução } \\
\text { semelhante em sua incidência } \\
\text { (de } 6,9 \text { para } 1,0 \text { e de } 7,8 \text { para } \\
1,4 \text { / 1.000 dias de ventilação, } \\
\text { em cada UTI). }\end{array}$ \\
\hline $\begin{array}{l}\text { Shirit P e col. } \\
\qquad 2015^{30} \\
\text { Israel }\end{array}$ & $\begin{array}{l}\text { Intervention to } \\
\text { reduce ventilator- } \\
\text { associated } \\
\text { pneumonia in } \\
\text { individuals on long- } \\
\text { term ventilation by } \\
\text { introducing a } \\
\text { customized bundle }\end{array}$ & $\begin{array}{c}\text { J Am } \\
\text { Geriatr Soc } \\
\text { IA1 }\end{array}$ & $\begin{array}{l}\text { Estudo de intervenção não } \\
\text { randomizado. }\end{array}$ & \begin{tabular}{|} 
Avaliar a eficácia de \\
um bundle de medidas \\
preventivas para reduzir \\
a PAV nos pacientes \\
em uso crônico de \\
ventiladores em \\
instalações de cuidados \\
prolongados.
\end{tabular} & $\begin{array}{c}\text { A taxa de PAV diminuiu de } \\
5,97 \text { antes da intervenção para } \\
2,34 \text { após a intervenção por } \\
\text { 1.000 dias de VM }(P<0,001) \text {. } \\
\text { O uso de antibióticos de } \\
\text { amplo espectro diminuiu de } \\
\text { 1.788 doses diárias antes da } \\
\text { intervenção para } 1.093 .\end{array}$ \\
\hline $\begin{array}{c}\text { Azab SF e } \\
\text { col. } \\
2015^{31} \\
\text { Egito }\end{array}$ & $\begin{array}{l}\text { Reducing } \\
\text { ventilator- } \\
\text { associated } \\
\text { pneumonia in } \\
\text { neonatal intensive } \\
\text { care unit using } \\
\text { "VAP prevention } \\
\text { Bundle": a cohort } \\
\text { study }\end{array}$ & $\begin{array}{c}\text { BMC Infect } \\
\text { dis./A2 }\end{array}$ & Estudo prospectivo. & $\begin{array}{c}\text { Avaliar a eficácia do } \\
\text { bundle de prevenção } \\
\text { na redução da PAV } \\
\text { na unidade de terapia } \\
\text { intensiva neonatal } \\
\text { (UTIN). }\end{array}$ & $\begin{array}{c}51 \% \text { dos pacientes } \\
\text { desenvolveram PAV no período. } \\
\text { A taxa de PAV/1.000 dias de } \\
\text { VM foi reduzida de } 67,8 \% \\
\text { (42/62) correspondendo a } 36,4 \\
\text { episódios na fase I, para } 38,2 \% \\
\text { (31/81) correspondendo a } 23 \\
\text { episódios após implementação } \\
\text { do bundle (fase II). }\end{array}$ \\
\hline $\begin{array}{c}\text { Sachetti A e } \\
\text { col. } \\
2014^{32} \\
\text { Brasil }\end{array}$ & $\begin{array}{l}\text { Adherence to the } \\
\text { items in a bundle } \\
\text { for the } \\
\text { prevention } \\
\text { of ventilator- } \\
\text { associated } \\
\text { pneumonia }\end{array}$ & $\begin{array}{l}\text { Rev. bras } \\
\text { ter Intensiva } \\
\text { /B2 }\end{array}$ & $\begin{array}{c}\text { Estudo quantitativo, descritivo } \\
\text { e de corte transversal. }\end{array}$ & $\begin{array}{c}\text { Avaliar a adesão ao } \\
\text { bundle de ventilação } \\
\text { mecânica em uma UTI, } \\
\text { bem como o impacto } \\
\text { dessa adesão nas taxas } \\
\text { de PAV. }\end{array}$ & $\begin{array}{l}\text { A taxa de adesão dos } \\
\text { profissionais foi de } 66,7 \% \text { após } \\
\text { intervenção. A incidência de } \\
\text { PAV foi semelhante em ambos } \\
\text { os períodos da pesquisa, } \\
\text { sendo } 28,5 / 1.000 \text { pacientes/ } \\
\text { dia na pré-intervenção e } \\
27,1 / 1.000 \text { pacientes/dia na } \\
\text { pós-intervenção. }\end{array}$ \\
\hline
\end{tabular}




\begin{tabular}{|c|c|c|c|c|c|}
\hline $\begin{array}{l}\text { Al-thaqafy MS } \\
\text { e col. } \\
2014^{33} \text { Arábia } \\
\text { Saudita }\end{array}$ & $\begin{array}{l}\text { Association of } \\
\text { compliance of } \\
\text { ventilator bundle } \\
\text { with incidence } \\
\text { of ventilator- } \\
\text { associated } \\
\text { pneumonia and } \\
\text { ventilator utilization } \\
\text { among critical } \\
\text { patients over } 4 \\
\text { years }\end{array}$ & $\begin{array}{l}\text { Ann Thorac } \\
\text { Med./A2 }\end{array}$ & $\begin{array}{l}\text { Estudo prospectivo e } \\
\text { transversal. }\end{array}$ & $\begin{array}{c}\text { Validar a associação do } \\
\text { bundle com a taxa de } \\
\text { PAV em um ambiente } \\
\text { tradicionalmente alto } \\
\text { de PAV e examinar } \\
\text { sua associação com a } \\
\text { utilização do ventilador. }\end{array}$ & $\begin{array}{c}\text { A adesão ao bundle do } \\
\text { ventilador aumentou de } 90 \% \\
\text { para } 97 \% \text {; a taxa de PAV } \\
\text { diminuiu de } 3,6 \text { (por } 1.000 \text { dias } \\
\text { de ventilação) para } 1,0 \text { e a } \\
\text { taxa de utilização do ventilador } \\
\text { diminuiu de } 0,73 \text { para } 0,59 .\end{array}$ \\
\hline $\begin{array}{c}\text { Dosher W e } \\
\text { col. } \\
2014^{34} \\
\text { Estados } \\
\text { Unidos }\end{array}$ & $\begin{array}{l}\text { The effect of } \\
\text { a nurse-led } \\
\text { multidisciplinary } \\
\text { team on } \\
\text { ventilator- } \\
\text { associated } \\
\text { pneumonia rates }\end{array}$ & $\begin{array}{l}\text { Critical } \\
\text { Care } \\
\text { Research } \\
\text { and } \\
\text { Practice } \\
\text { /B2 }\end{array}$ & Estudo retrospectivo. & \begin{tabular}{|c|} 
Examinar o efeito \\
de uma equipe de \\
prevenção de PAV \\
liderada por enfermeiros \\
sobre as taxas de PAV \\
em uma UTI médica \\
aberta em um hospital- \\
comunidade.
\end{tabular} & $\begin{array}{c}\text { A taxa média de PAV foi de } \\
3,20 / 1.000 \text { dias de ventilação } \\
\pm 5,71 \text { desvio padrão. } \\
\text { Redução média mensal na } \\
\text { taxa de PAV de } 0,27 / 1.000 \\
\text { dias de ventilação, } \square<0,001 \\
\text { (IC: }-0,40-0,13)\end{array}$ \\
\hline $\begin{array}{l}\text { Pérez-Granda } \\
\text { MJ e col. } \\
2014^{35} \\
\text { Espanha }\end{array}$ & $\begin{array}{l}\text { Impact of four } \\
\text { sequential } \\
\text { measures on } \\
\text { the prevention } \\
\text { of ventilator- } \\
\text { associated } \\
\text { pneumonia in } \\
\text { cardiac surgery } \\
\text { patients }\end{array}$ & $\begin{array}{l}\text { Crit Care/ } \\
\text { A1 }\end{array}$ & $\begin{array}{l}\text { Estudo prospectivo } \\
\text { ecológico. }\end{array}$ & $\begin{array}{c}\text { Avaliar o impacto } \\
\text { de quatro medidas } \\
\text { implementadas } \\
\text { sequencialmente para } \\
\text { prevenir } \\
\text { PAV em uma grande UTI } \\
\text { de cirurgia cardíaca. }\end{array}$ & \begin{tabular}{|} 
As taxas de PAV (episódios \\
$\quad / 1.000$ dias VM) antes \\
e durante a intervenção \\
foram: 23,9 versus 13,5, \\
respectivamente. O ${ }^{\circ}$ médio \\
de dias de VM / 1.000 dias \\
de internação foi de 507 \\
versus 375. O custo da \\
terapia antimicrobiana foi de \\
$€ 70.612$ versus $€ 52.775$. \\
Redução na taxa de PAV para \\
$41 \%$. Redução da taxa de \\
mortalidade para $10,2 \%$.
\end{tabular} \\
\hline $\begin{array}{c}\text { Eom JS e col. } \\
2014^{36} \text { Coreia } \\
\text { do Sul }\end{array}$ & $\begin{array}{l}\text { The impact of a } \\
\text { ventilator bundle } \\
\text { on preventing } \\
\text { ventilator- } \\
\text { associated } \\
\text { pneumonia: a } \\
\text { multicenter study }\end{array}$ & $\begin{array}{l}\text { Am J Infect } \\
\text { Control } \\
\text { IA2 }\end{array}$ & Estudo quase experimental. & $\begin{array}{c}\text { Analisar a taxa de PAV } \\
\text { em UTIs após o início } \\
\text { do bundle de PAV em } \\
\text { comparação com a taxa } \\
\text { de PAV nos últimos } 8 \\
\text { meses. }\end{array}$ & $\begin{array}{c}\text { A implementação do bundle } \\
\text { reduziu a taxa de PAV/1.000 } \\
\text { dias de VM, de 4,08 a 1,16 } \\
\text { casos. A taxa de densidade } \\
\text { de incidência foi de } 0,28 \text { para } \\
0,27 \text {. }\end{array}$ \\
\hline $\begin{array}{l}\text { Lim KO e col. } \\
2013^{37} \text { Taiwan }\end{array}$ & $\begin{array}{c}\text { Efficacy of } \\
\text { ventilator-associated } \\
\text { pneumonia } \\
\text { care bundle for } \\
\text { prevention of } \\
\text { ventilator-associated } \\
\text { pneumonia in the } \\
\text { surgical intensive } \\
\text { care units of a } \\
\text { medical center. }\end{array}$ & $\begin{array}{l}\text { J Microbiol } \\
\text { Immunol } \\
\text { Infect } \\
\text { IA1 }\end{array}$ & Estudo retrospectivo. & $\begin{array}{c}\text { Analisar a utilização do } \\
\text { ventilador e a incidência } \\
\text { de PAV entre janeiro de } \\
2006 \text { e março de } 2013 \\
\text { para avaliar o impacto } \\
\text { do bundle de PAV em } \\
\text { cinco UTI's cirúrgicas } \\
\text { no Hospital Nacional da } \\
\text { Universidade de Taiwan. }\end{array}$ & $\begin{array}{c}\text { A incidência de PAV diminuiu } \\
\text { de } 13,63 \text { para } 3,94 / 1.000 \\
\text { pacientes em uso de } \\
\text { ventilador e a sua densidade } \\
\text { diminuiu notavelmente de } \\
3,3 \text { para } 1,4 \text { casos por } 1.000 \\
\text { dias de VM. A utilização do } \\
\text { ventilador diminuiu de } 1148,5 \\
\text { para } 956,1 \text { dias de ventilador. }\end{array}$ \\
\hline $\begin{array}{l}\text { Ding S e } \\
\text { col. } 2013^{38} \\
\text { Estados } \\
\text { Unidos }\end{array}$ & $\begin{array}{l}\text { Temporal Trends } \\
\text { of ventilator- } \\
\text { associated } \\
\text { pneumonia } \\
\text { incidence and } \\
\text { the effect of } \\
\text { implementing } \\
\text { health-care } \\
\text { bundles in a } \\
\text { suburban } \\
\text { community. }\end{array}$ & Chest/A1 & $\begin{array}{l}\text { Estudo de coorte } \\
\text { retrospectivo de base } \\
\text { populacional. }\end{array}$ & $\begin{array}{c}\text { Mensurar as tendências } \\
\text { na incidência e no } \\
\text { resultado da PAV } \\
\text { antes e depois da } \\
\text { implementação do } \\
\text { bundle de PAV. }\end{array}$ & $\begin{array}{c}\text { A taxa de PAV permaneceu } \\
\text { igual e sem alteração com o } \\
\text { bundle. Estimativas anuais da } \\
\text { incidência de PAV variaram } \\
\text { entre } 7,1 \text { e } 10,4 \text { casos por } \\
1.000 \text { dias de ventilação, } \\
\text { com uma incidência ajustada } \\
\text { por idade de } 3,1 \text { vs } 5,6 \text { por } \\
100.000 \text { habitantes. }\end{array}$ \\
\hline
\end{tabular}




\begin{tabular}{|c|c|c|c|c|c|}
\hline $\begin{array}{l}\text { Alsadat R e } \\
\text { col. } \\
2012^{39} \\
\text { Síria }\end{array}$ & $\begin{array}{l}\text { Use of ventilator } \\
\text { associated } \\
\text { pneumonia bundle } \\
\text { and statistical } \\
\text { process control } \\
\text { chart to decrease } \\
\text { VAP rate in Syria. }\end{array}$ & $\begin{array}{l}\text { Avicenna J } \\
\text { med./C }\end{array}$ & $\begin{array}{l}\text { Estudo transversal, } \\
\text { prospectivo. }\end{array}$ & $\begin{array}{c}\text { Implementar o bundle } \\
\text { de PAV como um } \\
\text { projeto de melhoria } \\
\text { do desempenho nas } \\
\text { unidades de cuidados } \\
\text { intensivos para todos os } \\
\text { pacientes sob ventilação } \\
\text { mecânica, com o } \\
\text { objetivo de diminuir as } \\
\text { taxas de PAV. }\end{array}$ & $\begin{array}{c}\text { A adesão ao bundle aumentou } \\
\text { de } 33 \text { para } 80 \% \text { no hospital } 1 \text {, } \\
\text { de } 33 \text { para } 86 \% \text { no hospital } \\
2 \text { e de } 83 \text { para } 100 \% \text { no } \\
\text { hospital } 3 . \text { Em } 1.000 \text { dias de } \\
\text { ventilação, diminuição nas } \\
\text { taxas de PAV de } 30 \text { para } 6,4 \\
\text { no hospital } 1 \text {, de } 12 \text { para } 4,9 \\
\text { no hospital } 3 \text {, sem diminuir no } \\
\text { hospital } 2 .\end{array}$ \\
\hline $\begin{array}{c}\text { Viana WN e } \\
\text { col. } \\
2013^{40} \text { Brasil }\end{array}$ & $\begin{array}{l}\text { Ventilator- } \\
\text { associated } \\
\text { pneumonia } \\
\text { prevention by } \\
\text { education and two } \\
\text { combined } \\
\text { bedside strategies. }\end{array}$ & $\begin{array}{l}\text { Inte J Qual } \\
\text { health care/ } \\
\text { B1 }\end{array}$ & $\begin{array}{l}\text { Ensaio clínico não } \\
\text { controlado. }\end{array}$ & $\begin{array}{c}\text { Reduzir as taxas } \\
\text { de incidência de } \\
\text { pneumonia associada } \\
\text { à ventilação mecânica } \\
\text { (PAV) por meio de um } \\
\text { programa de prevenção } \\
\text { racional. }\end{array}$ & $\begin{array}{l}\text { A incidência de PAV diminuiu. } \\
\text { A média mensal antes da } \\
\text { intervenção foi de } 18,6 \pm 7,8 \\
/ 1.000 \text { dias de ventilação, } \\
\text { diminuindo para } 11,8 \pm \\
7,8 / 1.000 \text { dias de ventilação } \\
\text { após as intervenções. }\end{array}$ \\
\hline $\begin{array}{c}\text { Guanche- } \\
\text { Garcell H e } \\
\text { col. } \\
2013^{41} \\
\text { Cuba }\end{array}$ & $\begin{array}{l}\text { Effectivenes of a } \\
\text { multidimensional } \\
\text { approach for } \\
\text { the prevention } \\
\text { of ventilator- } \\
\text { associated } \\
\text { pneumonia in an } \\
\text { adult intensive } \\
\text { care unit in Cuba: } \\
\text { Findings of the } \\
\text { Internacional } \\
\text { Nosocomial } \\
\text { Infection Control } \\
\text { Consortium } \\
\text { (INICC). }\end{array}$ & $\begin{array}{c}\text { J Infect } \\
\text { and Public } \\
\text { Health/ } \\
\text { A1 }\end{array}$ & $\begin{array}{l}\text { Estudo prospectivo pré-pós- } \\
\text { vigilância. }\end{array}$ & \begin{tabular}{|c|} 
Avaliar o efeito \\
da abordagem \\
multidimensional \\
desenvolvida pelo \\
International Nosocomial \\
Infection Control \\
Consortium (INICC) \\
na redução das taxas \\
de PAV em pacientes \\
internados em uma UTI \\
adulto.
\end{tabular} & $\begin{array}{l}\text { A taxa basal de PAV foi de } \\
52,63 \text { por } 1.000 \text { dias de } \\
\text { ventilação, enquanto no } \\
\text { período de intervenção a taxa } \\
\text { foi de } 15,32 \text { por } 1.000 \text { dias } \\
\text { de ventilação. Houve uma } \\
\text { redução na taxa de PAV de } \\
70 \% \text {. }\end{array}$ \\
\hline $\begin{array}{c}\text { Mehta Y e col. } \\
2013^{42} \\
\text { India }\end{array}$ & $\begin{array}{l}\text { Effectiveness of a } \\
\text { multidimensional } \\
\text { approach for } \\
\text { prevention of } \\
\text { ventilator- } \\
\text { associated } \\
\text { pneumonia in } 21 \\
\text { adult intensive-care } \\
\text { units from } 10 \text { cities } \\
\text { in India: findings } \\
\text { of the International } \\
\text { Nosocomial } \\
\text { Infection Control } \\
\text { Consortium } \\
\text { (INICC). }\end{array}$ & $\begin{array}{l}\text { Epidemiol } \\
\text { Infect/A2 }\end{array}$ & $\begin{array}{l}\text { Estudo de coorte prospectivo } \\
\text { e quase experimental. }\end{array}$ & \begin{tabular}{|c|} 
Relatar o efeito \\
da abordagem \\
multidimensional do \\
International Nosocomial \\
Infection Control \\
Consortium (INICC) \\
para a redução da PAV \\
em pacientes adultos \\
hospitalizados.
\end{tabular} & $\begin{array}{l}\text { A taxa basal de PAV foi } \\
\text { de 17,43 / } 1.000 \text { dias de } \\
\text { ventilação mecânica, e } \\
\text { 10,81 durante a intervenção, } \\
\text { mostrando uma redução de } \\
\text { 38\% da PAV. }\end{array}$ \\
\hline $\begin{array}{c}\text { Caserta RA } \\
\text { e col. } \\
2012^{43} \\
\text { Brasil }\end{array}$ & $\begin{array}{l}\text { A program } \\
\text { for sustained } \\
\text { improvement } \\
\text { in preventing } \\
\text { ventilator } \\
\text { associated } \\
\text { pneumonia in an } \\
\text { intensive care } \\
\text { setting }\end{array}$ & $\begin{array}{l}\text { BMC Infec } \\
\text { Dis/A2 }\end{array}$ & Estudo quase experimental. & \begin{tabular}{|c|} 
Avaliar se a \\
implementação \\
sustentada do bundle \\
de PAV na UTI poderia \\
efetivamente reduzir \\
a incidência de \\
pneumonia associada \\
à ventilação mecânica \\
(PAV).
\end{tabular} & $\begin{array}{c}\text { A densidade de incidência de } \\
\text { PAV por } 1.000 \text { dias de VM foi } \\
\text { de } 1,3 \text { em } 2009 \text { (10.889) e } 2 \\
\text { em } 2010 \text { (11.095). Nos meses } \\
\text { em que houve adesão de } \\
\text { 95\% ao bundle de ventilação, } \\
\text { obteve-se zero incidência de } \\
\text { PAV. }\end{array}$ \\
\hline
\end{tabular}




\begin{tabular}{|c|c|c|c|c|c|}
\hline $\begin{array}{c}\text { Sedwick e col. } \\
2012^{44} \\
\text { Estados } \\
\text { Unidos }\end{array}$ & $\begin{array}{l}\text { Using evidence- } \\
\text { based practice to } \\
\text { prevent ventilator } \\
\text { associated } \\
\text { pneumonia. }\end{array}$ & $\begin{array}{c}\text { Critical } \\
\text { Care Nurse/ } \\
\text { A1 }\end{array}$ & $\begin{array}{c}\text { Estudo } \\
\text { colaborativomulticêntrico. }\end{array}$ & \begin{tabular}{|} 
Desenvolver um \\
bundle de ventilação \\
e práticas de cuidados \\
para enfermeiros em \\
unidades de terapia \\
intensiva para reduzir \\
a taxa de pneumonia \\
associada à ventilação \\
mecânica.
\end{tabular} & $\begin{array}{c}\text { A adesão às práticas foram } \\
\text { superiores à } 90 \% \text {. As taxas } \\
\text { PAV diminuíram de } 9,47 \\
\text { para } 1,9 \text { casos por } 1.000 \\
\text { dias de ventilação. Houve } \\
\text { uma economia estimada de } \\
\text { aproximadamente US } \$ 1,5 \\
\text { milhão. }\end{array}$ \\
\hline $\begin{array}{c}\text { J. Rello e col. } \\
2012^{45} \\
\text { Espanha }\end{array}$ & $\begin{array}{l}\text { A care bundle } \\
\text { approach for } \\
\text { prevention } \\
\text { of ventilator- } \\
\text { associated } \\
\text { pneumonia }\end{array}$ & $\begin{array}{c}\text { Clin } \\
\text { Microbiol } \\
\text { Infect/A1 }\end{array}$ & $\begin{array}{c}\text { Estudo de coorte colaborativo } \\
\text { multicêntrico. }\end{array}$ & $\begin{array}{c}\text { Determinar o impacto da } \\
\text { implementação de um } \\
\text { bundle de cuidados para } \\
\text { a prevenção de PAV nas } \\
\text { taxas de PAV e duração } \\
\text { da ventilação mecânica. }\end{array}$ & $\begin{array}{l}\text { O cumprimento de todas as } \\
\text { medidas após a intervenção } \\
\text { foi }<30 \%(264 / 885) \text {. Apesar } \\
\text { disso, a incidência de PAV } \\
\text { diminuiu de } 15,5 \%(23 / 149) \\
\text { para } 11,7 \%(104 / 885) .\end{array}$ \\
\hline $\begin{array}{l}\text { Arroliga AC } \\
\text { e col. } \\
2012^{46} \\
\text { Estados } \\
\text { Unidos }\end{array}$ & $\begin{array}{l}\text { Reduction in } \\
\text { the Incidence } \\
\text { of Ventilator- } \\
\text { Associated } \\
\text { Pneumonia: A } \\
\text { Multidisciplinary } \\
\text { Approach. }\end{array}$ & $\begin{array}{l}\text { Respir } \\
\text { Care/A1 }\end{array}$ & $\begin{array}{l}\text { Estudo de coorte } \\
\text { retrospectivo. }\end{array}$ & $\begin{array}{c}\text { Relato do processo } \\
\text { implementado em uma } \\
\text { instituição por uma } \\
\text { força-tarefa focada na } \\
\text { redução da PAV. Os } \\
\text { pesquisadores relataram } \\
\text { o processo que este } \\
\text { grupo implementou } \\
\text { desde abril de } 2007 \text { e } \\
\text { compararam as taxas } \\
\text { de PAV na população } \\
\text { adulta nos anos de } 2008 \\
\text { e } 2009 .\end{array}$ & $\begin{array}{l}\text { A taxa de PAV em } 2008 \text { foi de } \\
4,3 \text { enquanto em } 2009 \text { foi de } \\
1,2 \text { (por } 1.000 \text { dias de VM). A } \\
\text { mortalidade hospitalar foi de } \\
26,1 \% \text { e não houve diferença } \\
\text { entre os dois anos. A adesão } \\
\text { ao bundle ventilatório foi de } \\
92 \% \text {. A adesão ao cuidado oral } \\
\text { melhorou de } 33 \% \text { para } 97 \% \text {. }\end{array}$ \\
\hline $\begin{array}{l}\text { Pogorzelska, } \\
\text { M e col. } \\
2011^{47} \\
\text { Estados } \\
\text { Unidos }\end{array}$ & $\begin{array}{l}\text { Impact of the } \\
\text { ventilator bundle } \\
\text { on ventilator- } \\
\text { associated } \\
\text { pneumonia } \\
\text { in intensive care } \\
\text { unit. }\end{array}$ & $\begin{array}{l}\text { Int J Health } \\
\text { Care/B1 }\end{array}$ & Levantamento transversal. & \begin{tabular}{|c} 
Examinar a adoção \\
dos elementos do \\
bundle de ventilação; \\
determinar a eficácia de \\
elementos individuais \\
e definir características \\
na redução da PAV; \\
determinar a eficácia \\
de dois elementos \\
específicos de \\
infecção na redução \\
da PAV; e, avaliar \\
os efeitos cruzados \\
do cumprimento dos \\
elementos nas infecções \\
da corrente sanguínea \\
associadas ao acesso \\
central.
\end{tabular} & \begin{tabular}{|} 
A taxa média geral de PAV \\
para 279 UTIs foi de 2,67 \\
por 1.000 dias de VM. $68 \%$ \\
das UTIs tinham protocolo \\
de ventilação, e apenas $66 \%$ \\
dessas UTIs monitoram sua \\
implementação. $39 \%$ relatam \\
conformidade total do bundle \\
como $95 \%$ do tempo ou mais. \\
Os elementos individuais \\
ou apenas um dos dois \\
elementos relacionados à \\
infecção não teve impacto \\
na PAV. Houve associação \\
entre o cumprimento de dois \\
elementos infecciosos e taxas \\
menores.
\end{tabular} \\
\hline $\begin{array}{c}\text { Bird D e col. } \\
2010^{48} \\
\text { Estados } \\
\text { Unidos }\end{array}$ & $\begin{array}{l}\text { Adherence } \\
\text { to Ventilator- } \\
\text { Associated } \\
\text { Pneumonia } \\
\text { Bundle and } \\
\text { Incidence of } \\
\text { Ventilator- } \\
\text { Associated } \\
\text { Pneumonia in the } \\
\text { Surgical Intensive } \\
\text { Care Unit. }\end{array}$ & $\begin{array}{c}\text { Archives of } \\
\text { surgery } \\
\text { IA1 }\end{array}$ & Estudo prospectivo. & $\begin{array}{l}\text { Examinar o impacto da } \\
\text { adesão a um bundle de } \\
\text { PAV sobre a incidência } \\
\text { de PAV em unidades } \\
\text { de terapia intensiva } \\
\text { cirúrgica (UTICs). }\end{array}$ & $\begin{array}{l}\text { O cumprimento do bundle de } \\
\text { PAV aumentou ao longo do } \\
\text { período de estudo de } 53 \% \\
\text { e } 63 \% \text { para } 91 \% \text { e } 81 \% \text { em } \\
\text { cada respectiva UTI. A taxa de } \\
\text { PAV diminuiu de } 10,2 \text { para } 3,4 \\
\text { casos por } 1.000 \text { dias de VM. } \\
\text { Estimada uma economia de } \\
\text { custos de US } \$ 1,08 \text { milhão. }\end{array}$ \\
\hline
\end{tabular}




\begin{tabular}{|c|c|c|c|c|c|}
\hline $\begin{array}{c}\text { Perugini MRE } \\
\text { e col. } 2015^{49} \\
\text { Brasil }\end{array}$ & $\begin{array}{l}\text { Impacto de um } \\
\text { bundle nas taxas } \\
\text { de pneumonia } \\
\text { associada à } \\
\text { ventilação } \\
\text { mecânica (PAV) } \\
\text { em uma unidade } \\
\text { de terapia intensiva } \\
\text { pediátrica em } \\
\text { Londrina-PR. }\end{array}$ & $\begin{array}{c}\text { Semina: } \\
\text { Ciências } \\
\text { Biológicas e } \\
\text { da Saúde/ } \\
\text { B5 }\end{array}$ & $\begin{array}{l}\text { Estudo transversal, de } \\
\text { intervenção. }\end{array}$ & $\begin{array}{c}\text { Analisar o efeito do } \\
\text { pacote de intervenções } \\
\text { de controle da infecção, } \\
\text { a educação, as taxas } \\
\text { de PAV na Unidade } \\
\text { Pediátrica de Terapia } \\
\text { Intensiva (UTIP) do } \\
\text { Hospital Universitário de } \\
\text { Londrina-PR. }\end{array}$ & $\begin{array}{c}\text { Comparando-se o período } \\
\text { pré-intervenção com o pós- } \\
\text { intervenção, a taxa média } \\
\text { de PAV foi de } 49,6 \% \text { e } \\
17,5 \% \text {, respectivamente, } \\
\text { demonstrando uma redução } \\
\text { de } 64,8 \% \text { na mesma. }\end{array}$ \\
\hline $\begin{array}{l}\text { Pulzi Júnior, } \\
\text { AS e col. } \\
2015^{50} \\
\text { Brasil }\end{array}$ & $\begin{array}{l}\text { Pneumonia } \\
\text { associada à } \\
\text { ventilação } \\
\text { mecânica como } \\
\text { indicador de } \\
\text { qualidade e } \\
\text { segurança em } \\
\text { saúde. }\end{array}$ & $\begin{array}{l}\text { Rev méd } \\
\text { Minas } \\
\text { Gerais/B3 }\end{array}$ & $\begin{array}{l}\text { Estudo retrospectivo, } \\
\text { descritivo e comparativo. }\end{array}$ & $\begin{array}{c}\text { Analisar o indicador de } \\
\text { resultado (incidência da } \\
\text { PAV) concomitante à } \\
\text { análise do indicador de } \\
\text { processo, adesão aos } \\
\text { componentes do bundle } \\
\text { de PAV, procurando } \\
\text { correlação entre estes. }\end{array}$ & $\begin{array}{l}\text { Adesão ao bundle mostrou-se } \\
\text { inferior ao esperado: decúbito } \\
\text { elevado = } 62 \% \text {; higiene oral } \\
58 \% \text {; despertar diário }=34 \% \text {. } \\
\text { Houve redução na mediana } \\
\text { da densidade de incidência } \\
\text { de PAV }(2011=17,86 ; 2012= \\
11,95 ; 2013=5,88) .\end{array}$ \\
\hline
\end{tabular}

Fonte: Elaborado pelos autores, 2019.

*UTI= Unidade de Terapia Intensiva

${ }^{*} \mathrm{PAV}=$ Pneumonia Associada à Ventilação

*VM= Ventilação Mecânica

A execução de bundles simplificados, ou seja, com menos itens do que os propostos pelo $\mathrm{IHI}$, também se mostram capazes de reduzir as taxas de PAV e isso pode estar associado ao diferente impacto de componentes individuais( ${ }^{(56)}$, assim como adesão de $85 \%$ ao tratamento oral que resultou em redução de $72 \%$ da ocorrência de $\operatorname{PAV}^{(21)}$. Comparando uma metanálise publicada em 2013, foi observado que os cuidados orais foram atribuídos a uma redução de $40 \%$ nos casos de $\mathrm{PAV}^{(57)}$. A higiene oral é claramente importante na prevenção da PAV, devido ao fato de que os patógenos responsáveis eram também encontrados na cavidade ora|(58-59). $O$ que remete também a importância da aspiração das secreções, e mostra a inclusão do uso de um tubo endotraqueal com drenagem de secreção subglótica no bundle ${ }^{(23)}$ ou em conjunto com o mesmo ${ }^{(43)}$. $O$ uso deste tubo, comparado ao uso do tubo endotraqueal padrão, pode apresentar como vantagens o atraso no desenvolvimento da PAV e a diminuição na incidência da mesma ${ }^{(23,35)}$, além de possivelmente estar associado a redução do tempo de $\mathrm{VM}^{(60)}$. O estudo de Marini e col. $(2016)^{(61)}$, mostra esta associação, onde todos os pacientes foram intubados com o tubo endotraqueal com sucção subglótica e isso refletiu na diminuição da incidência de PAV(61). Em contrapartida, nem sempre as diferenças se apresentam significativas, quando se analisa que $16,3 \%$ dos pacientes entubados com tubo endotraqueal com drenagem de secreção subglótica desenvolveram $\mathrm{PAV}$, comparado à $35,5 \%$ que foram entubados com o tubo padrão e também a desenvolveram ${ }^{(20)}$.Além disso, esse tipo de material requer gastos financeiros maiores, o que acaba colocando como um item não obrigatório( ${ }^{(36)}$.

Dentre alguns fatores de risco para pacientes em
UTI, estão o grande número de prescrições de antibióticos $^{(62-63)}$, nutrição enteral| ${ }^{(64-65)}$ e traqueostomia ${ }^{(26,65-66)}$. Evidencia-se que o bundle é capaz de agir diretamente na diminuição do uso de antibióticos ${ }^{(28,30)}$, e alcançar redução da PAV entre pacientes traqueostomizados ${ }^{(26)}$. Com a diminuição da PAV, mais pacientes podem se alimentar via oral e diminuir as chances de aspiração em decorrência da sonda nasogástrica ${ }^{(30)}$.

Apesar dos já comprovados benefícios do bundle, ainda é difícil implementá-lo e mantê-lo em uma adesão adequada, requerendo engajamento e conhecimento da equipe ${ }^{(29,45)}$. Alguns fatores podem contribuir para sustentar a adesão, como: seleção de um profissional para supervisionar a implementação do bundle e cooperação entre a equipe de UTI e de controle de infecção ${ }^{(33)}$. Cenários onde a adesão não é expressiva, pode levar à não diferença significativa nas taxas de $\mathrm{PAV}^{(32,67)}$.Aspectos dificultam a análise destas taxas, como a subjetividade na definição da $P A V^{(68)}$ e a possível interferência disso em seu diagnóstico ${ }^{(48)}$.

Por fim, ressalta-se que o impacto do bundle, se apresenta positivo não só para as taxas de $\mathrm{PAV}$, pois influencia na diminuição do tempo de internação( ${ }^{(27)}$, do uso da ventilação mecânica ${ }^{(33)}$ na redução dos custos hospitalares $^{(28,44)}$ e diminuição da mortalidade ${ }^{(49)}$.

Estudo realizado recentemente sobre cuidados preventivos para pneumonia associados a ventilação mecânica salientou a importância da capacitação dos profissionais da saúde para uma melhor assistência, bem como, a necessidade de realizar ações preventivas para redução das infecções do trato respiratório e do tempo de internação do paciente ${ }^{(69)}$. 


\section{CONCLUSÕES}

Diante da análise dos estudos, evidencia-se um expressivo número de benefícios após a implementação do bundle, principalmente na redução das taxas de PAV. $O$ bundle sugerido pelo $\mathrm{IHI}$ possui medidas de fácil acesso e baixos custos financeiros, que se aplicadas de forma objetiva provocam um grande efeito para os pacientes, equipe e instituição de saúde. A modificação da ferramenta pelas unidades hospitalares, também se mostrou eficaz. Através desta revisão integrativa, salientou-se de forma considerável a dificuldade de adesão às medidas por parte dos profissionais, o que traz a necessidade de estudos para buscar compreender quais os obstáculos mais presentes através da perspectiva da equipe. 


\section{REFERÊNCIAS}

I. Agência Nacional de Vigilância Sanitária (BR). Critérios Diagnósticos de Infecções Relacionadas à Assistência à Saúde/Agência Nacional de Vigilância Sanitária. Brasília:Anvisa, 2017[citado em 10 set. 2018]. Disponível em: https:// www20.anvisa.gov.br/segurancadopaciente/index.php/publicacoes/item/criterios-diagnosticos-das-infeccoes-relacionadas-a-assistencia-a-saude?category_id=191.

2. Silva SG, Nascimento ERP, Salles, RK. Bundle to prevent ventilator-associated pneumonia: a collective constructi. Text Context Nursing. [Internet]. 2012 Out/Dez[citado em 09 out. 20 I8];2I (4): 837-44. Disponível em: http://www. scielo.br/pdf/tce/v2In4/en_I4.pdf.

3. Carvalho CRR. Ventilator-associated pneumonia. J bras pneumol. [Internet]. 2006 Jul/Ago [citado em 10 set. 2018],32(4). 6 p. Disponivel em: http://www.jornaldepneumologia.com.br/detalhe_artigo.asp?id=1254.

4. Cunha FA, Oliveira AFS. Complicações decorrentes da ventilação mecânica ao paciente de unidades de terapia intensiva (uti) adulto. Scientific Journal [Internet]. $2018 \mathrm{Jan} /$ Mar[citado em II set. 20I8];I(I):I38-6I. Disponível em: http://healthresearchjournal.com/hrj/index.php/hrj/article/ view/28/10.

5. Gonçalves E. Nursing care in the prevention of ventilator -Associated pneumonia (vap) in intensive care unit, an Integrative review of the literature. Scientific Journal [Internet] 20 I 8 jan/Mar[citado em II set 20 I8]; I I ): I23-37. Disponível em: http://healthresearchjournal.com/hrj/index.php/hrj/ article/view/27/9.

6. Institute for Healthcare Improvement. Prevent How-to Guide: Prevent Ventilator-Associated Pneumonia. Cambridge: Institute for Healthcare Improvement; 20 I2[citado em I5 set. 2018]. Disponível em: https://www.chpso.org/sites/ main/files/file-attachments/ihi_howtoguidepreventvap.pdf.

7. Scottish Intensive Care Society Audit Group. VAP prevention bundle: guidance for implementation. 2008[citado em 09 set. 2018]. Disponível em: https://www.sicsag.scot.nhs. uk/hai/VAP-Prevention-Bundle-web.pdf.

8. Hellyer TP, Ewan V,Wilson P, Simpson AJ. The Intensive Care Society recommended bundle of interventions for the prevention of ventilator-associated pneumonia.J Intensive Care Soc. [Internet]. 20 I6[citado em I 3 nov. 20 I8]; I7(3):238-43. Doi: doi.org/I0.1 I77/I75I|437/664446I.

9. Lerma FA, Garcia MS, Lorente L, Añon JM, Álvarez J, e col.. Guidelines for the prevention of ventilator-associated pneumonia and their implementation. The spanish "Zero-VAP" bundle. Med intensiva [internet]. 2014 Mai[citado 10 dez. 2018];38(4):226-36 Disponível em: Doi: doi.org/10.1016/j. medin.2013.12.007

10. Agência Nacional de Vigilância Sanitária (BR). Medidas de prevenção de infecção relacionada à assistência à saúde. Brasília:ANVISA; 2017.

I I. Almeida AS, Aragão NRO, Moura E, Lima GC, Hora EC,
Silva LASM. Sentimentos dos familiares em relação ao paciente internado na unidade de terapia intensiva. Rev. bras. enferm. [Internet]. $2009 \mathrm{Dec}$ [cited $2020 \mathrm{Apr}$ I8]; 62(6): 844-849. Available from: http://www.scielo.br/scielo.php?script=sci_arttext\&pid=S0034=7 | 672009000600007-\&lngen. https://doi.org/I0.I590/S0034-7I672009000600007.

12. Vieira CENK, Andrade OS, Enders BC, Coura AS, Dutra MOM. Acciones de enfermería para la prevención de la neumonía asociada a ventilación mecánica: revisión sistemática. Enfermería Global [Internet]. 2014 Jul[citado 13 dez. 20।8];35:338-49. Disponível em: http://scielo.isciii.es/ pdf/eg/v13n35/revision4.pdf.

13. Ramos NOS, Alves GR. Importância dos cuidados da equipe de enfermagem na prevenção da pneumonia associada a ventilação mecânica (pavm): revisão da literatura. Revista Científica Fac Mais [Internet]. 2018[citado em 23 nov. 20I8];XIII(I):3I-8. Disponível em: http://revistacientifica.facmais.com.br/wp-content/uploads/20I8/06/2.-A -IMPORT\%C3\%82NCIA-DOS-CUIDADOS-DA-EQUIPE-DE-ENFERMAGEM-NA-PREVEN\%C3\%87\%C3\%83ODA-PNEUMONIA-ASSOCIADA-A-VENTILA\%C3\%87\%C3\%83O-MEC\%C3\%82NICA-PAVM-REVIS\%C3\%83O-DA -LITERATURA.pdf.

14. Brasil. Lei n. 7.498 de junho de 1986. Dispõe sobre a regulamentação do exercício da enfermagem, e dá outras providências. [Internet]. I986[citado em 12 nov. 20 I8]. Disponível em: http://www.planalto.gov.br/ccivil_03/LEIS/L7498.htm.

15. Mendes KD, Silveira RCCP, Galvão CM. Revisão integrativa: método de pesquisa para a incorporação de evidências na saúde e na enfermagem. Texto Contexto Enferm. [Internet]. 2008 Out/Dez[citado em 17 out. 20I8];I7( 4):758-64. Disponível em: http://www.scielo.br/pdf/tce/vI7n4/I8.pdf.

16. Camelo Silvia Helena Henriques. Competência profissional do enfermeiro para atuar em Unidades de Terapia Intensiva: uma revisão integrativa. Rev. Latino-Am. Enfermagem [Internet], 2012 Feb [cited 2020 Apr I8]; 20(I): 192-200. Available from: http://www.scielo.br/scielo.php?script=sci_ arttext\&pid=SOI04-I I6920 I $2000100025 \&$ Ing=en. https:// doi.org/I0.1590/S0I04-II6920I2000100025.

17. Ercole FF, Melo LS, Alcoforado CLGC. Revisão integrativa versus revisão sistemática. Rev Min Enferm. [Internet]. 20 I4[citado em I5 out. 20 I8];8(I):9- I2. Doi: I0.5935/I4I52762.2014000I.

18. Al-abdely HM, Khidir YM, Rosenthal VD, Orellano PW, Alazhary M, Kaid E, e col.. Impact of the International Nosocomial Infection Control Consortium (INICC)'s multidimensional approach on rates of ventilator-associated pneumonia in intensive care units in 22 hospitals of 14 cities of the Kingdom of Saudi Arabia. J Infect Public Health [Internet]. 20 I 8 Sep/Oct [citado em 23 nov. 20 I 8]; I I (5), 677-84. Doi: doi.org/10.1016/j.jiph.2018.06.002.

19. Álvarez-Lerma F, Paloma-Martinez M, Sánchez-Garcia M, Martinez-Alonso M, Álvarez-Rodriguez J, Lorente I, e col. 
Prevention of ventilator-associated pneumonia: the multimodal approach of the spanish icu "pneumonia zero" program. Crit Care Med [Internet]. 2017[citado em I 5 fev. 2019];46(2): I81-88.

20. Burja S, Belec T, Bizjak N, Mori J, Markota A, Sinkovic A. Efficacy of a bundle approach in preventing the incidence of ventilator associated pneumonia (vap). Bosn J Basic Med Sci [Internet]. 2017[citado em I5 fev. 20 I9]; I8(I): I05-09. Doi: doi.org/10.17305/bjbms.2017.2278.

2I. Su KC, Kou YR, Lin FC, Wu CH, Huang SF, Shiung TF, col. A simplified prevention bundle with dual hand hygiene audit reduces early-onset ventilator-associated pneumonia in cardiovascular surgery units: an interrupted time-series analysis. PloS One [Internet]. 2017[citado em I5 fev. 2019];12(8):I-I8. Doi: doi.org//0.|37//journal. pone.0182252.

22. Haque A, Riaz Q, Ali SA. Implementation of ventilator bundle in pediatric intensive care unit of a developing country. J Coll Physicians Surg Pak [Internet]. 20 I7[citado em 16 fev. 2019];27(5):3 I6-18. Disponível em: https://www.ncbi.nlm. nih.gov/pubmed/28599698.

23. Akdogana O, Ersoy Y, Kuzucu C, Gedik E, Togai T, Yetkin F. Assessment of the effectiveness of a ventilator associated pneumonia prevention bundle that contains endotracheal tube with subglottic drainage and cuff pressure monitorization. Braz J Infect Dis. [Internet]. 2017[citado em I6 fev. 20I9]; 2I(3):276-8I. Doi: doi.org/10.10I6/j. bjid.2017.01.002.

24. Mogyorodi B, Dunai E, Gál J, Zsolt I. Ventilator-associated pneumonia and the importance of education of ICU nurses on prevention - preliminary results, Interv Med Appl Sci. [Internet]. 2016[citado em I6 f2v. 2019];8(4):|47-5I. Doi: doi.org//0.1556/1646.8.2016.4.9.

25. Rodrigues AN, Fragoso LVC, Beserra FM, Ramos IC. Determining impacts and factors in ventilator-associated pneumonia bundle. Rev. Bras. Enferm. [online]. [Internet]. 2016[citado em 16 fev. 2019];69( 6):II08-14. Doi: doi. org/I0.1590/0034-7I67-2016-0253.

26. Peña-López Y, Pujol M, campins M, González-Antelo A, Rodrigo JÁ, Balcells J, col. Implementing a care bundle approach reduces ventilator-associated pneumonia and delays ventilator-associated tracheobronchitis in children: differences according to endotracheal or tracheostomy devices. Int J Infect Dis. [Internet]. 2016 Nov[citado em 16 fev 2018];52:43-8.

27. Parisi M, Gerovasili V, Dimopoulos S, Kampisiouli E, Goga C, Privolioti $\mathrm{E}$, col. Use of ventilator bundle and staff education to decrease ventilator associated pneumonia in intensive care patients. Crit Care Nurse. [Internet]. 2016[citado em 2 fev. 20l9];36(5):I-7.

28. Ferreira CR, Souza DF, Cunha TM, Tavares M, Reis SSA, Pedroso RS, e col.The effectiveness of a bundle in the prevention of ventilator-associated pneumonia. The Braz J Infect
Dis [Internet]. 2016[citado em 22 fev, 2019];20(3):267-7I. Doi: doi.org/10.1016/j.bjid.2016.03.004.

29. Daniel M, Booth M, Ellis k, Maher S, Lingmate A. Details behind the dots: How different intensive care units used common and contrasting methods to prevent ventilator associated pneumonia. BMJ Quallmprov Rep [Internet]. 2015 Mar[citado em 23 fev. 20 I9];4(I):u207660.w3069. Doi: doi. org/l 0.I I36/bmjquality.u207660.w3069.

30. Shitrit P, Meirson M, Mendelson G, Chowers M. Intervention to reduce ventilator-associated pneumonia in individuals on long-term ventilation by introducing a customized bundle. J Am Geriatr Soc. [Internet].2015[citadoem 23 fev. 2019];63(I0):2089-93. Doi: doi.org/ I 0. I I I I/jgs. I 3646.

3I. Azab SF, Sherbiny HS, Saleh SH, Elsaeed WF, Elshafiey MM, Siam MA, e col. Reducing ventilator-associated pneumonia in neonatal intensive care unit using "vap prevention Bundle": a cohort study. BMC Infect dis. [Internet]. 20I5[citado em 23 fev. 20 I9]; I5(I):3 I4. Doi: doi.org/ I0.। I 86/s I 2879-0 I5- I062-I.

32. Sachetti A, rech V, Dias AS, Fontana C, Barbosa GL, Schlichting $D$. Adherence to the items in a bundle for the prevention of ventilator-associated pneumonia. Rev. bras. ter. intensiva [Internet]. 20 I4[citado em 25 fev. 20 I9];26(4):3559. Doi: doi.org/ I0.5935/0 I03-507X.20 I 40054.

33. Al-thaqafy MS, El-Saed A, Arabi YM, Balkhy HH. Association of compliance of ventilator bundle with incidence of ventilator-associated pneumonia and ventilator utilization among critical patients over 4 years. Ann Thorac Med. [Internet]. 2014 Out [citado em 25 fev. 2019];9(4)22I-6 Doi: doi.org/10.4103/1817-1737.140132.

34. Dosher W, Bradley e col. The effect of a nurse-led multidisciplinary team on ventilator-associated pneumonia rates. Critical Care Research and Practice [Internet]. 2014[citado 27 fev. 2019]:5p. Doi: doi.org/ / 0. I I55/20 I 4/68262 I.

35. Pérez-Granda MJ, Barrio JM, Muñoz P, Hortal J, Rincón c, Bouza E. Impact of four sequential measures on the prevention of ventilator-associated pneumonia in cardiac surgery patients. Crit Care [Internet]. 2014[citado em 10 fev. 2019]; /8(2):R53. Disponível em: https://www.ncbi.nlm.nih. gov/pubmed/246670I I.

36. EOM JS, Lee MS, Chun HK, Choi HJ, Jung SY, Kim YS, col. The impact of a ventilator bundle on preventing ventilator-associated pneumonia: a multicenter study. Am J Infect Control [Internet]. 2014[citado em 27 fev. 20I9];42(I):347. Doi: doi.org/10.1016/j.ajic.2013.06.023.

37. Lim KP, Kuo SW, Ko WJ, Sheng WH, Chang YY, Hong MC, col. Efficacy of ventilator-associated pneumonia care bundle for prevention of ventilator-associated pneumonia in the surgical intensive care units of a medical center. J Microbiol Immunol Infect. [Internet]. 20I5[citado em 27 fev. 2019];48(3):3 I62 I. Doi: doi.org/I0. I0 I6/j.jmii.20 I3.09.007.

38. Ding S, Kilickaya O, Senkal S, Gajic O, Hubmary RD, e col. Temporal trends of ventilator-associated pneumonia incidence and the effect of implementing health-care bundles 
in a suburban community. Chest. [Internet]. 2013[citado em 23 fev. 2019];|44(5):|46I-68. Doi: doi.org/I0.1378/ chest. I2-1675.

39. Alsadat R, Al-Bardan H, Malzloum MN, Shamah AA, Marie A, Dakkar A, e col. Use of ventilator associated pneumonia bundle and statistical process control chart to decrease vap rate in Syria. Avicenna J med. [Internet]. 2012[citado em 27 fev. 2019];2(4)79-83. Doi: doi.org/I0.4I03/223I0770.110736.

40. Viana WN, Bragazzi C, Castro JEC,Alves MB, Rocco JR.Ventilator-associated pneumonia prevention by education and two combined bedside strategies. Inte J Qual health carea. [Internet]. 2013 Jul [citado 27 fev. 2019];25(3):308- I3. Doi: doi.org// 0.1093/intqhc/mzt025.

4I. Guanche-Garcell H, Morales-Pérez C, Rosenthal VD. Effectiveness of a multidimensional approach for the prevention of ventilator-associated pneumonia in an adult intensive care unit in Cuba: findings of the International Nosocomial Infection Control Consortium (INICC). J Infect Public Health [Internet]. 20I3[citado em 08 mar. 20I3];6(2):98-I07. Doi: doi.org/10.1016/j.jiph.2012.11.009.

42. Mehta Y, Jaggi N, Rosenthal VD, Rodrigues C, Todi SK, Saini $\mathrm{N}$, Udwadia FE, e col. Effectiveness of a multidimensional approach for prevention of ventilator-associated pneumonia in 21 adult intensive-care units from 10 cities in India: findings of the International Nosocomial Infection Control Consortium (INICC). Epidemiol Infect. [Internet] 20I3[citado em 08 mar. 20I9]; I4I(I2):2483-9I. Doi: doi. org/I0.10I7/S09502688I300038I.

43. Caserta RA, Marra AR, Durão MS, Silva CV, Santos OFP, Neves HS, e col. A program for sustained improvement in preventing ventilator associated pneumonia in an intensive care setting. BMC Infect Dis [Internet]. 2012 Set[citado em 08 mar 2019]; I2(I):234. Doi: doi.org/10.I I86/147/-2334-12-234.

44. Sedwick MB, Smith-Lance M, Reeder SJ, Nardi J. Using evidence-based practice to prevent ventilator-associated pneumonia. Critical Care Nurse [Internet]. 2012[citado 03 mar. 20 I9];32(4):4 I-5I. Doi: doi.org/ 0.4037/ccn20 I 2964.

45. Rello J, Afonso E, Lisboa T, Ricart M, Balsera B, Rovira A, e col. A care bundle approach for prevention of ventilator-associated pneumonia. Clin Microbiol Infect. [Internet] 2013[citado em 03 mar. 2019];19(4): 363-9. Doi: doi.org/I0. I I I //j. I469-069I.2012.03808.x.

46. Arroliga AC, Pollard CL, Wilde CD, Pellizzari SJ, Chebbo A, Song J, col. Reduction in the incidence of ventilator-associated pneumonia: a multidisciplinary approach. Respir Care. [Internet]. 2012 [citado em 20 fev. 2019];57(5):688-96. Doi: doi.org/10.4187/respcare.01392 .

47. Pogorzelska, m, Stone PW, Furuya EY, Perencevich EM, Larson EL, Goldmann D, Dick A. Impact of the ventilator bundle on ventilator-associated pneumonia in intensive care unit. Int J Health Care [Internet]. 201 I [citado em 02 mar. 2019];23(5):538-44. Doi: doi.org/10.1093/intqhc/mzr049.
48. Bird D, Zambuto A, O’Donell C, Silva J, Korn C, Burke R, col. Dorothy e col. Adherence to ventilator-associated pneumonia bundle and incidence of ventilator-associated pneumonia in the surgical intensive care unit. Arc Surg [Internet]. 2010[citado em 03 mar. 2019]; | 45(5):465-70. Doi: doi.org/10.1001/archsurg.2010.69.

49. Perugini MRE, Perugini VH, Figueira FD, Fontana LMS, Diniz JJ,Santos DL, col. Impacto de um bundle nas taxas de pneumonia associada à ventilação mecânica (PAV) em uma unidade de terapia intensiva pediátrica em Londrina-PR. Semina: Ciências Biológicas e da Saúde [Internet]. 20I5[citado em 03 mar. 20I9];36(I supl):259-66. Doi: 10.5433/1679-0367.2014v35n2p259.

50. Pulzi Júnior, AS, Ferraz RRN, Lapchick MS. Pneumonia associada à ventilação mecânica como indicador de qualidade e segurança em saúde. Rev méd Minas Gerais [Internet]. 2019 Jan[citado em 24 mar. 2019];25(4):5 I 7-22, 20I5. Doi: doi.org/10.5935/2238-3182.201501 I6.

5I. Rosenthal VD, Guzman S, Crnich C. Impact of an infection control program on rates of ventilator-associated pneumonia in intensive care units in 2 Argentinean hospitals. Am J Infect Control [Internet]. 2006 Mar[citado 10 abr. 2019];34(2):58-63. Disponível em: https://www.ncbi.nlm. nih.gov/pubmed/I 6490607.

52. Tao L, Hu B, Rosenthal VD, Zhang Y, Gao X, He L. Impact of a multidimensional approach on ventilator-associated pneumonia rates in a hospital of Shanghai: findings of the International Nosocomial Infection Control Consortium. J Crit Care [Internet]. 2012 Out [citado em $23 \mathrm{fev}$. 2019];27(5):440-6. Doi: doi: 10.1016/j.jcrc.201 I.12.018.

53. Leblebicioglu H,Yalcin AN, Rosenthal VD, Koksal I, Sirmatel F, Unal S, col. Effectiveness of a multidimensional approach for prevention of ventilator-associated pneumonia in $1 \mathrm{I}$ adult intensive care units from 10 cities of Turkey: findings of the International Nosocomial Infection Control Consortium (INICC). Infection [Internet]. $2013 \mathrm{Abr}$ [citado em 30 mar. 20 I9];4 I (2):447-56. Doi: doi: I0. I007/s I 50 I0-0 I3-0407-I.

54. Augustyn B. Ventilator-associated pneumonia: risk factors and prevention. Crit Care Nurse [Internet]. 2007 Ago [citado 10 abr. 2019]; 27(4):32-6.

55. Cocanour CS, Peninger M, Domonoske BD, Li T, Wright $B$, Valdivia A, col. Decreasing ventilator associated pneumonia in a trauma ICU. J Trauma. 2006 Jul; 61:I22-29. Doi: 10.1097/0I.ta.0000223971.25845.b3.

56. Collard HR, Saint S, Matthay MA. Prevention of ventilator associated pneumonia: an evidence-based systematic review.Ann Intern Med. 2003 Mar; 138(6):494-50I.

57. Hua F, Xie H,Worthington H, Furness S, Zhang Q, col. Oral hygiene care for critically ill patients to prevent ventilator -associated pneumonia. Cochrane Database of Systematic Review. 2016. Disponível em: https://www.cochranelibrary. com/cdsr/doi//0.1002/I465 I858.CD008367.pub3/epdf/full. 58. Heo SM, Haase EM, Lesse AJ, Gill SR, Scannapieco FA. Ge- 
netic relationships between respiratory pathogens isolated from dental plaque and bronchoalveolar lavage fluid from patients in the intensive care unit undergoing mechanical ventilation. Clin Infect Dis. 2008 Dez;47(I2):I562-70. Doi: doi.org/I0.1086/593 I93 PMID: I899|508.

59. Oliveira LCBS, Carneiro PPM, Fischer RG, Tinoco EMB. A presença de patógenos respiratórios no biofilme bucal de pacientes com pneumonia nosocomial. Revista Brasileia de Terapia Intensiva [Internet]. 2007 Out/Dez[citado 20 jan. 2019];19(4):428-33. Disponível em: http://www.scielo.br/pdf/rbti/v19n4/a04v19n4.pdf.

60. Muscedere J, Rewa O, McKechnie K, Jiang X, Laporta D, Heyland DK: Subglottic secretion drainage for the prevention of ventilator-associated pneumonia:a systematic review and meta-analysis. Crit Care Med. 20I I Aug;39(8): I985-9I. Doi: doi: 10.1097/CCM.0b013e318218a4d9.

61. Marini AL, Khan R, Mundekkadan S. Multifaceted bundle interventions shown effective in reducingVAP rates in our multidisciplinary ICUs. BMJ Qual Improv Rep. 2016 Abr;5(I): pii: u205566.w2278. Doi: 10. I 136/bmjquality.u205566.w2278.

62. Craven DE. Preventing ventilator-associated pneumonia in adults: sowing seeds of change. Chest. 2006 Jul; I 30(I):25 I-60.

63. Avarnitis M, Anagnostou T, Kourkoumpetis TK, Ziakas PD, Desalermos A, Mylonakis E. The impact of antimicrobial resistance and aging in VAP outcomes: experience from a large tertiary care center. PLoS ONE. 20।4;9(2): e89984. Doi: 10.1371/journal.pone.0089984.
64. Vincent JL. Critical care-where have we been and where are we going? Crit Care. 2013;17(Suppl. I): S2-12. Doi: 10.1 I86/cc|l500.

65. Apostolopoulou E, Bakakos P, Katostaras T, Gregorakos L. Incidence and risk factors for ventilator-associated pneumonia in 4 multidisciplinary intensive care units in Athens, Greece. Respir Care. 2003 Jul;48(7):68I-8.

66. Ibrahim EH,Tracy L, Hill C, FraserVJ, Kollef MH.The occurrence of ventilator-associated pneumonia in a community hospital: risk factors and clinical outcomes. Chest. 200I Ago; I 20(2):555-6I. DOI: 10.1378/chest. I 20.2.555.

67. Bloos F, Muller S, Harz A, Gugel M, Geil D, Egerland K, col. Effects of staff training on the care of mechanically ventilated patients:A prospective cohort study. Br J Anaesth. 2009 Ago; 103(2):232-7 Doi: 10.1 I86/cc I I500.

68. Klompas M, Khan Y, Kleinman K, Evans RS, Lloyd JF, Stevenson $K$, et al. Multicenter evaluation of a novel surveillance paradigm for complications of mechanical ventilation. PLoS One. 20I I Mar;6(3):el8062. Doi: I0.137I/journal.pone.00I8062.

69. Cabral BG, Celestino Júnior AF, Santana ME, Matos ECO. Cuidados preventivos para pneumonia associada a ventilação mecânica: revisão integrativa. Revista Enfermagem Atual In Derme. 2020; 9 I(29): I 3 I- I 40.

Recebido: 2019-07-22

Aceito: $2020-02-20$ 